\title{
tic\&société
}

Vol. 12, $\mathrm{N}^{\circ} 1$ | 1er semestre 2018

Communs numériques et communs de la connaissance

\section{Le permaculteur et son robot: les microfermes et la gouvernance des nouvelles technologies}

\section{Peter HANAPPE}

\section{OpenEdition}

\section{Journals}

Édition électronique

URL : http://journals.openedition.org/ticetsociete/2452

DOI : 10.4000/ticetsociete. 2452

\section{Éditeur}

Association ARTIC

\section{Édition imprimée}

Pagination : 229-253

\section{Référence électronique}

Peter HANAPPE, «Le permaculteur et son robot : les microfermes et la gouvernance des nouvelles technologies », tic\&société [En ligne], Vol. 12, № 1 | 1er semestre 2018, mis en ligne le 31 mai 2018, consulté le 10 octobre 2020. URL : http://journals.openedition.org/ticetsociete/2452 ; DOI : https:// doi.org/10.4000/ticetsociete.2452 
tic\&société - 12(1), 2018

\title{
Le permaculteur et son robot: les microfermes et la gouvernance des nouvelles technologies
}

\author{
Peter HANAPPE
}

Peter Hanappe a fait des études en électronique et en informatique à l'Université de Gand, en Belgique. À Paris, il réalise un doctorat sur les environnements informatiques pour la composition et la synthèse sonore en temps réel à l'Ircam (Centre George Pompidou, Paris). Peu après, il rejoint le Sony Computer Science Laboratory à Paris, où il travaille initialement sur de nouveaux modes de création et de diffusion de contenus impliquant la participation des communautés en ligne. II se concentre ensuite sur des projets dans le domaine du développement durable. II a participé à la mise en place d'un système collectif pour cartographier le bruit en milieu urbain (NoiseTube) et a optimisé des composants essentiels pour les simulations climatiques à grande échelle en collaboration avec le Met Office du Royaume-Uni et I'Université d'Oxford. II travaille actuellement sur le thème des systèmes alimentaires durables et communautaires et, en tant qu'informaticien, au rôle que les technologies peuvent jouer dans de tels systèmes. Cependant, il est conscient que la technologie ne peut résoudre qu'une partie de l'équation, c'est pourquoi il s'intéresse beaucoup au concept de Commons pour traiter des nombreuses questions socioéconomiques. hanappe@csl.sony.fr 


\section{Le permaculteur et son robot : les microfermes et la gouvernance des nouvelles technologies}

Résumé : Les licences libres sont généralement considérées comme une garantie suffisante contre les risques que peuvent présenter les outils non libres. Les œuvres intellectuelles couvertes par ces licences font partie des biens communs informationne/s. L'argument principal de ce texte est que ces licences ne sont pas suffisantes pour assurer un équilibre entre les concepteurs des technologies et les utilisateurs finaux. Dans ce texte, nous nous intéressons particulièrement à l'agriculture. Dans ce domaine, nous assistons aujourd'hui à une intégration entre les TIC, l'intelligence artificielle et la robotique. L'impact qu'auront ces systèmes est difficile à évaluer. Pour accompagner l'introduction des nouvelles technologies, nous suggérons de prendre en compte un autre commun: celui des pratiques agricoles. Nous proposons d'encadrer l'introduction des nouvelles technologies agricoles par des systèmes participatifs de garantie tels que mis en place par des labels participatifs.

Mots-clés : robotique, agroécologie, microfermes, gouvernance, pratiques agricoles.

Abstract: Free and Open Source licenses are generally considered a sufficient guarantee against the risks of closedsource technologies. The tools protected by open licenses belong to what is called the knowledge Commons. The main argument of this text is that these licenses are insufficient to ensure a balance between the producers of new technologies and their users. We focus on the case of agriculture. This field is currently witnessing a fast integration of ICT, artificial intelligence and robotics, and the impact of these changes is hard to predict. However, to assist with the introduction of these new technologies and manage their impact, we propose using Participative Systems of Guarantee, such as those employed by existing participative quality labels.

Keywords: robotics, agroecology, micro-farms, governance, agricultural practices. 
Le permaculteur et son robot :

les microfermes et la gouvernance des nouvelles technologies

Resumen: Las licencias de software libres o de software de código abierto son consideradas generalmente como una garantía suficiente contra los riesgos que podrían presentar las herramientas no libres. Las obras intelectuales cubiertas por estas licencias forman parte de los bienes comunes informacionales. El argumento principal de este texto es que las licencias no son suficientes para asegurar un equilibrio entre los diseñadores de tecnologías y los usuarios finales. Se basa particularmente en la agricultura, en donde hoy se asiste a una integración de las TIC, la inteligencia artificial y la robótica. Resulta muy difícil evaluar el impacto futuro de estos sistemas. Para acompañar la introducción de nuevas tecnologías, se sugiere tomar en cuenta otro bien común: las prácticas agrícolas. Finalmente se encuadran las nuevas tecnologías agrícolas con Sistemas Participativos de Garantía, como los sistemas establecidos por los labels participativos.

Palabras claves: robótica, agroecología, micro-granjas, gobernanza, prácticas agrícolas. 


\section{Peter HANAPPE}

\section{Introduction ${ }^{1}$}

Les technologies ont toujours joué un rôle important dans l'agriculture. Cela n'a jamais été aussi évident que dans la Révolution verte d'après-guerre où elles ont occupé une place prépondérante. Cette révolution a apporté une productivité et une sécurité alimentaire inédites dans notre histoire. Sa force de frappe a été la conséquence de l'utilisation des équipements motorisés, de la normalisation et de la spécialisation des techniques de production, notamment en monocultures, et de l'utilisation des pesticides et des fertilisants de synthèse. Malgré ses succès, l'agriculture traverse en ce moment une zone de turbulence. Les pratiques courantes sont remises en question et, en parallèle, la robotique et l'intelligence artificielle, soutenues par les technologies de l'information et de la communication (TIC), s'invitent à la campagne. La poussée des nouvelles technologies ne rassure pas tout le monde. On constate aujourd'hui un intérêt grandissant pour les microfermes qui font revivre d'anciennes techniques paysannes et qui défendent une approche agroécologique forte (voir plus bas). Elles prônent l'utilisation d'outils low-tech, en partie parce qu'elles considèrent qu'une vision technophile et productiviste a rendu l'agriculture industrielle insensible aux aspects environnementaux et sociaux.

Les licences libres sont souvent considérées comme une garantie suffisante contre les risques que peuvent présenter les outils technologiques, notamment contre la perte de contrôle des outils dont on dépend pour son travail. Les œuvres intellectuelles couvertes par ces licences sont généralement appelées des biens communs informationnels (knowledge commons, en anglais) (Hess et Ostrom, 2011). L'argument principal de ce texte est que ces licences ne sont pas suffisantes pour assurer un équilibre

\footnotetext{
${ }^{1}$ Je remercie mon collègue David Colliaux pour ses commentaires et ses corrections pendant la préparation du texte. Le projet Robotics for Microfarms (ROMI) bénéficie d'un financement du programme de recherche et innovation H2020 de l'Union européenne sous le Grant agreement $n^{\circ} 773875$.
} 
Le permaculteur et son robot :

les microfermes et la gouvernance des nouvelles technologies

entre ceux qui conçoivent les technologies, d'un côté, et ceux qui sont concernés par ces technologies, de l'autre. Effectivement, l'usage des nouvelles technologies, dans un champ d'activité donné, s'ajoute aux pratiques existantes dans ce métier. Ces pratiques sont, elles aussi, un bien commun. Ainsi, ces nouvelles technologies et leurs usages doivent être sélectionnés, définis et encadrés par les membres de cette communauté.

Nous proposons alors de réfléchir à la structure de gouvernance qui pourra réunir les concepteurs des nouvelles technologies et les communautés de pratiques à travers des groupes de travail mixtes. On pourra s'appuyer sur la notion des labels participatifs qui, depuis les années 1970, ont aidé à préserver les pratiques paysannes (IFOAM, 2008). Leur modèle de fermes - locales, familiales, agroécologiques - est parmi ceux qui résistent le mieux dans ces temps difficiles. Nous défendons, dans ce texte, que ce modèle de gouvernance participatif offre probablement le meilleur cadre de travail pour intensifier la recherche sur l'utilisation de la robotique pour les microfermes.

\section{Le contexte social}

La Révolution verte a apporté une grande productivité. Elle a aussi eu des conséquences néfastes (Carson, 1962 ; Gliessman, 2014) : les tracteurs sont d'une grande efficacité, mais causent la compaction du sol et émettent du $\mathrm{CO}_{2}$. Les engrais synthétiques apportent les nutriments nécessaires, mais une application trop abondante contribue à la pollution des eaux et à l'émission des gaz à effet de serre (FAO et ITPS, 2015). Les produits phytosanitaires maintiennent les maladies sous contrôle, mais diminuent la biodiversité et représentent un risque pour la santé. Les monocultures augmentent la productivité, mais, le sol se retrouvant sans couvert végétal, elles favorisent leur érosion (Pimentel, 2006).

La Révolution verte a eu d'autres effets négatifs - directs ou indirects. En soixante ans, la part de population active qui travaille dans l'agriculture est passée de $31 \%$, en 1955, à 3,1\% en 2008 


\section{Peter HANAPPE}

(Charroin et al., 2012) ${ }^{2}$. Comme d'autres secteurs, l'industrie agroalimentaire connaît une concentration économique croissante (Council of Economic Advisers, 2016 ; Fuglie et al., 2012 ; Shand, 2012). Cette situation a mis les agriculteurs dans une position précaire (Bouanchaud, 2016 ; Charroin et al., 2012). Pour la société, cette industrialisation a créé une distance entre la chaîne de production alimentaire et les consommateurs, ce qui expliquerait en partie l'augmentation des maladies liées aux mauvais régimes alimentaires (Pereira et al., 2005).

La filière agroalimentaire est consciente d'un grand nombre de ces problèmes et les conférences sur les solutions pour demain se multiplient. Les nouvelles technologies, sous les noms d'agriculture de précision ou d'agriculture numérique, suscitent beaucoup d'espoir quant à l'amélioration de la productivité et la diminution de l'impact environnemental (Bournigal et al., 2015). Nous assistons aujourd'hui à la transition des démonstrateurs avancés (Gonzalez-De-Santos et al., 2016) vers les premières solutions commerciales (voir Figure 2). Les tracteurs autonomes qui tracent leurs parcours tout seuls pendant que les drones cartographient les champs sont maintenant envisageables. Une analyse des images aériennes "dans le cloud" calcule ensuite des doses très précises d'engrais et de pesticides à appliquer (Mulla, 2013). Puis, les objets connectés suivent en temps réel les champs et les interventions des agriculteurs.

En parallèle de ces démarches, nous constatons qu'il existe également beaucoup d'expérimentations sur les techniques de culture alternatives. Ainsi, il n'existe plus une agriculture, mais des agricultures (Transrural, 2009). Plusieurs formes nous intéressent particulièrement ici: l'agroécologie forte, la permaculture et le développement des microfermes. Depuis les années 1980, Eliot Coleman et John Jeavons aux États-Unis, et plus récemment Jean-Martin Fortier au Canada, remettent à jour d'anciennes

\footnotetext{
2 II ne semble pas toujours évident de démêler la cause et l'effet de ce phénomène. La mécanisation a-t-elle entraîné la perte des emplois, ou l'exode rural vers les villes a-t-il accéléré l'adoption des outils motorisés ? Cette question est encore d'actualité. Certains fermiers s'opposent à une automatisation des tâches agricoles, car ils craignent la perte d'emploi, tandis que d'autres se plaignent d'un manque de main-d'œuvre et accueillent l'automatisation comme une solution.
} 
Le permaculteur et son robot :

les microfermes et la gouvernance des nouvelles technologies

techniques de maraîchage (souvent des techniques françaises). Ils cultivent un grand nombre de légumes sur des petites surfaces, parfois en mélangeant les variétés en polyculture, et ont principalement recours au travail manuel (Coleman, 1995 ; Fortier, 2012 ; Jeavons, 2012). Parallèlement, la permaculture, introduite par David Holmgren et Bill Mollison, définit un cadre conceptuel pour concevoir un style de vie écologique (Holmgren, 2002; Mollison et Holmgren, 1990). Leurs écrits ont d'abord fait écho dans les milieux qui visent l'autosuffisance alimentaire. Toujours dans les années 1980, des agronomes tel que Gliessman, Altieri et Holt-Giménez tirent les conclusions des dégâts environnementaux et sociaux provoqués par la Révolution verte en Amérique latine. De retour dans leurs universités américaines, ils élargissent la notion de l'agroécologie pour inclure l'étude des systèmes alimentaires complets et la positionnent comme un mouvement qui $œ u v r e$ pour une justice sociale pour les petites fermes. C'est cette variante que nous avons appelée l'agroécologie forte dans ce texte (Altieri, 1995 ; Gliessman, 2014 ; Holt-Giménez, 2006 ; Wezel et al., 2009).

Nous assistons aujourd'hui à une intégration de ces différents mouvements et pratiques. Les lieux, en France, qui en sont représentatifs incluent La Ferme du Bec Hellouin, Le Mas de Beaulieu et les Fermes d'Avenir (Figure 1). Ils représentent une rupture par rapport aux techniques classiques - y compris par rapport à l'agriculture biologique (Hervé-Gruyer et Hervé-Gruyer, 2014 ; Morel et Léger, 2015). Les microfermes prônent des méthodes a priori plus écologiques, favorisent l'utilisation d'outils low-tech (Hervé-Gruyer, 2015) et, selon les premières études, sont économiquement viables (Guégan et Léger, 2015). Elles constituent un sujet de recherche très riche tant sur le plan agronomique que socioéconomique. Cette évolution va de pair avec un intérêt renouvelé pour les différentes formes de vente directe et de circuits courts (Aubry et Chiffoleau, 2009). 


\section{Peter HANAPPE}
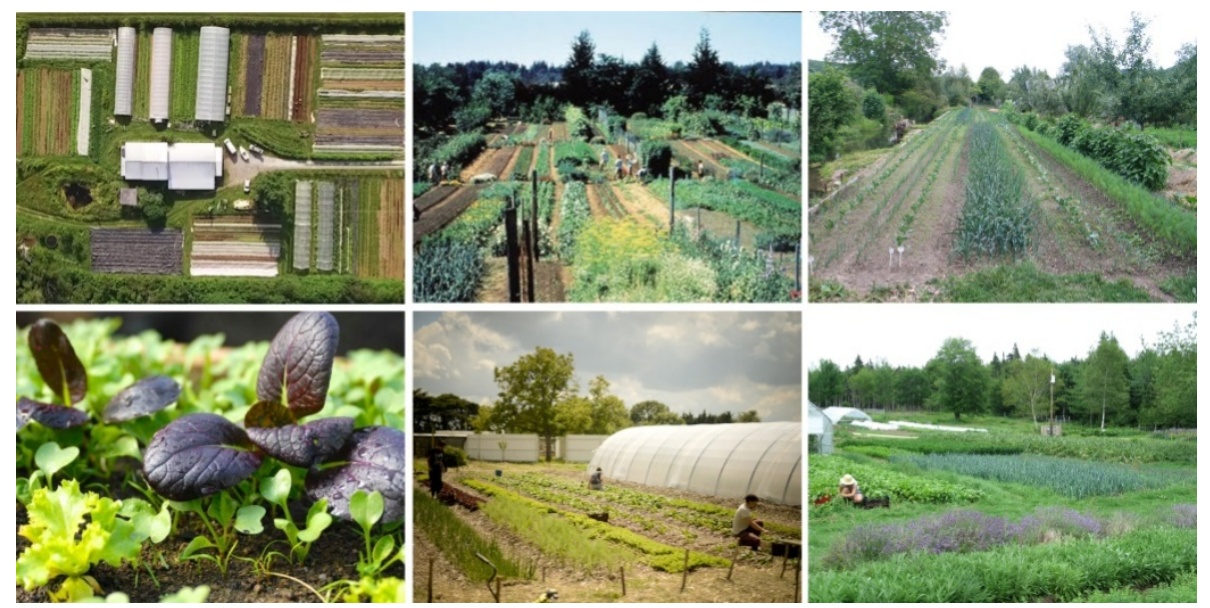

Figure 1. Quelques exemples de microfermes (de gauche à droite à partir du haut): Les jardins de la Grelinette (J.-M. Fortier, Canada); Grow Bio-intensive Garden (J. Jeavons, É.-U.) ; la Ferme du Bec Hellouin (P. et C. Hervé-Gruyer, France); détail de polyculture à Bec Hellouin ; Ferme de la Bourdaisière (M. de Rostolan, France) ; Four Seasons Farm (E. Coleman, É.-U.).

$\mathrm{Si}$, dans les pays économiquement avancés, l'agriculture intensive a favorisé les grandes exploitations, il existe néanmoins plus de $\mathbf{4 7 5}$ millions de fermes de moins de deux hectares dans le monde (Lowder, Skoet et Singh, 2014). Elles sont considérées comme des éléments clés pour la sécurité alimentaire de demain (De Schutter, 2014). En comparaison, globalement, " seulement » 27 millions de tracteurs sont en activité3.

\section{Le contexte technologique}

Les robots agricoles ne sont pas nouveaux. Plusieurs projets de recherche et commerciaux existent en France et ailleurs (Figure 2). Néanmoins, la plupart des projets visent une application dans des champs en monocultures.

\footnotetext{
${ }^{3}$ Voir: Nation Master (2000). Agriculture > Tractors: Countries Compared. Repéré à http://www.nationmaster.com/country-info/stats/Agriculture/Tractors
} 


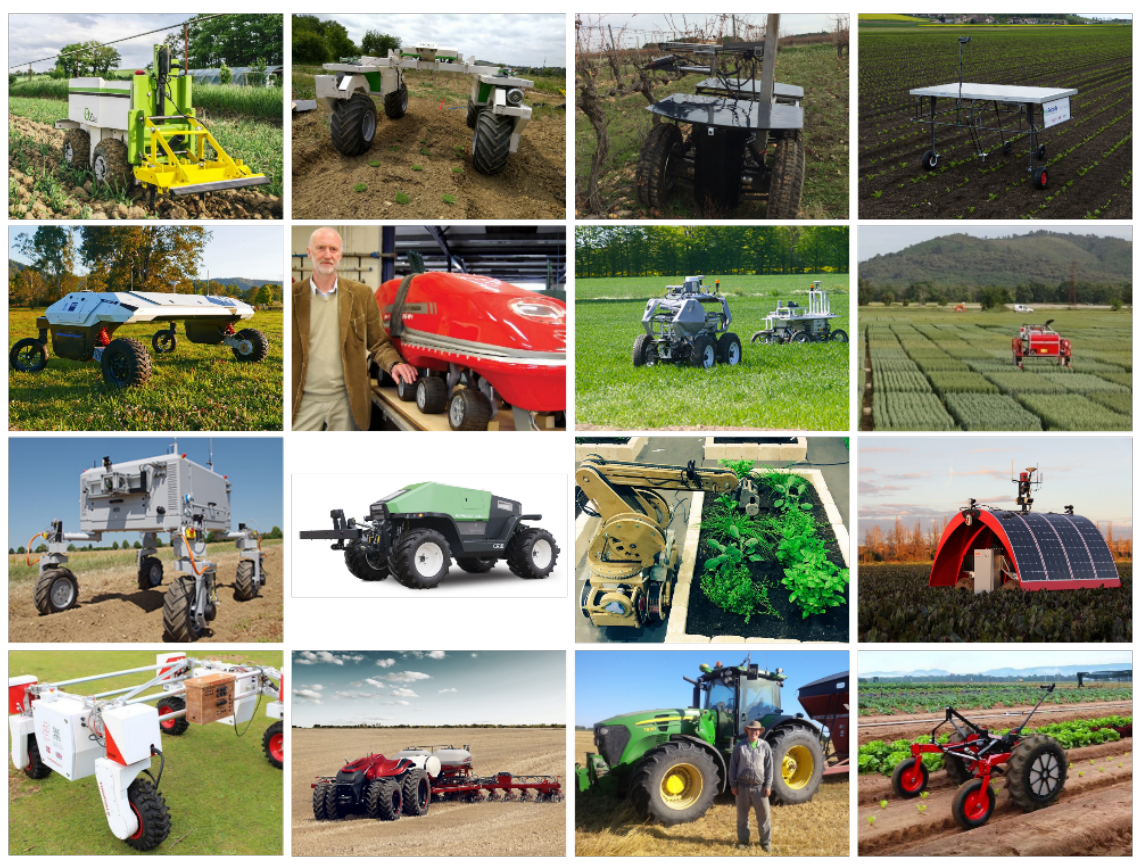

Figure 2. Une sélection de propositions pour l'agriculture robotiques (de gauche à droite à partir du haut) : Oz (Naïo Technologies, commercialisé); Dino (Naïo Technologies, commercialisé); MYCE (Wall-ye, commercialisé); ecoRobotix (commercialisé); AgBot II (Australian Centre for Robotics Vision, projet de recherche) ; Harper Adams University (projet de recherche) ; IRSTEA (projet de recherche); Phénomobile (HiPhen, commercialisé); BoniRob (Deepfield Robotics/Bosch, projet de recherche); Greenbot (Precision Makers, commercialisé); Bot2Karot (projet de recherche); Ladybird (University of Sydney, projet de recherche); Thorvalds (Norwegian University Of Life Sciences, projet de recherche); Case IH Autonomous Vehicle Concept (Case $\mathrm{IH})$; Open Source autonomous tractor (Matt Reiner); Digital Farmhand (Australian Centre for Field Robotics, projet de recherche).

Un projet qui aborde le problème différemment est FarmBot (Figure 3). Ce projet est ancré dans la culture DIY et fab lab, et cet angle d'attaque a permis de se débarrasser d'un certain nombre d'idées préconçues sur les robots existants. C'est un outil léger en aluminium, loin des engins classiques et lourds, en acier. Sa conception est en Open Hardware ${ }^{4}$ et utilise des composants

\footnotetext{
${ }^{4}$ Le code source et les plans sont accessibles à https://github.com/FarmBot et https://genesis.farm.bot/docs (dernière consultation le 9 avril 2018).
} 


\section{Peter HANAPPE}

standards, notamment des éléments qu'on trouve dans les imprimantes $3 \mathrm{D}$ et les fraiseuses numériques. Cela lui permet de proposer un prix qui est près de dix fois inférieur au prix des solutions concurrentes, mais, surtout, c'est un outil numérique qui permet de travailler de manière précise en trois dimensions et qui accepte une organisation spatiale des cultures sans contraintes. II gère aussi bien les polycultures que les monocultures. Les interventions mécaniques sont précises et demandent peu d'énergie. FarmBot propose une intégration complète avec un logiciel de gestion des cultures. Sa conception modulaire lui permet d'échanger les outils automatiquement et il peut semer, désherber, arroser et suivre des cultures par caméra. L'objectif de FarmBot est d'offrir un outil avancé aux particuliers pour la gestion d'un petit potager, de l'ordre de quelques mètres carrés. Sans doute, les agriculteurs n'y voient qu'un jouet, mais les points positifs soulevés ci-dessus ne sont pas restreints aux petites surfaces.

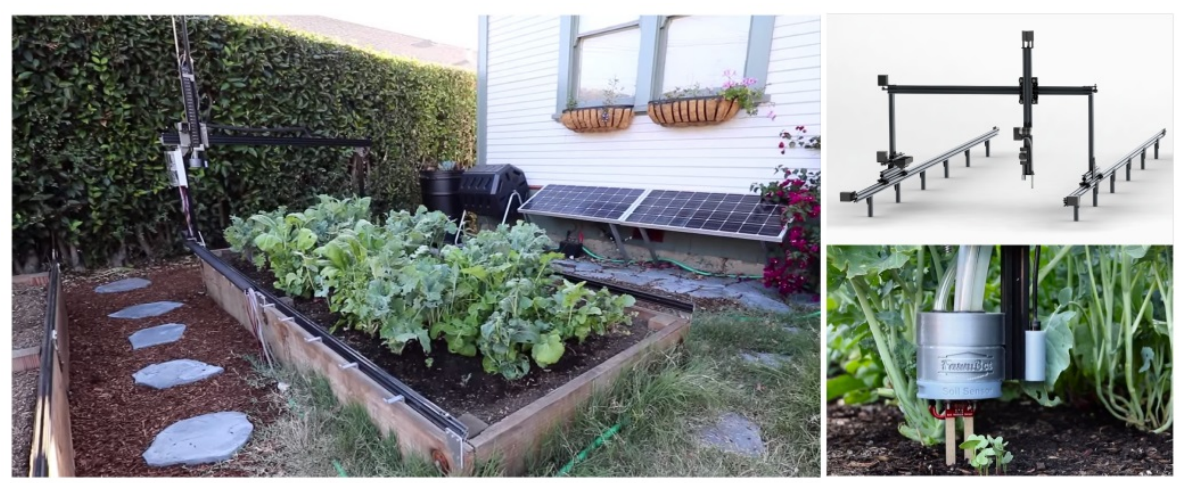

Figure 3. FarmBot utilise un contrôle numérique pour placer des outils (semoir, arrosoir, pince de désherbage, capteur d'humidité du sol) dans un espace de travail fixe. Source : https://genesis.farm.bot/docs

En 2016, nous avons démarré le développement d'un robot pour microfermes maraîchères nommé "LettuceThink ». La première application pratique que nous visons est le désherbage automatique. Dans le maraîchage biologique, cette tâche peut représenter jusqu'à $25 \%$ du temps de travail. Comme FarmBot, 
Le permaculteur et son robot:

les microfermes et la gouvernance des nouvelles technologies

nous avons choisi de modifier une fraiseuse numérique existante, en l'occurrence la fraiseuse X-Carve. Nous avons mis une bineuse rotative électrique à la place de la fraiseuse. Le tout est monté sur une structure avec des roues commandées numériquement (Figure 4). Nous avons ajouté des caméras et des ordinateurs à 30 euros, les Raspberry Pi. Le robot détecte les plants grâce à une caméra placée au-dessus du plan de travail. Le processus est détaillé à la Figure 5. Les premières expériences avec l'outil de désherbage en plein champ montrent qu'un passage deux fois par semaine permet de maîtriser le développement des adventices (Garlanda, 2017).
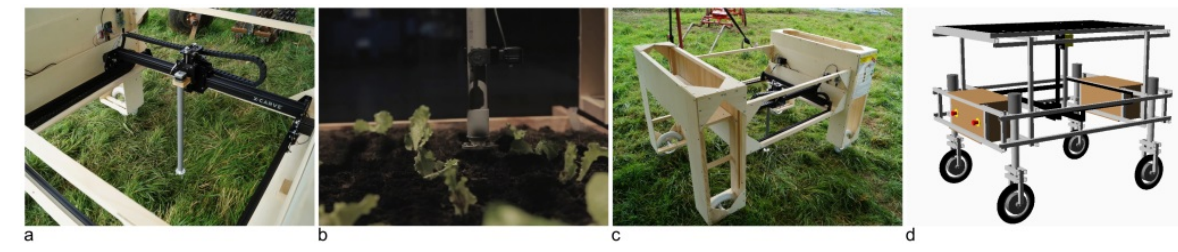

Figure 4. (a) LettuceThink utilise une fraiseuse numérique pour placer l'outil de désherbage dans le plan de travail. (b) Cet outil remue la couche superficielle du sol pour perturber la germination des mauvaises herbes. (c) Le premier prototype. (d) Le deuxième prototype prévu en 2017 pour une utilisation dans les champs.
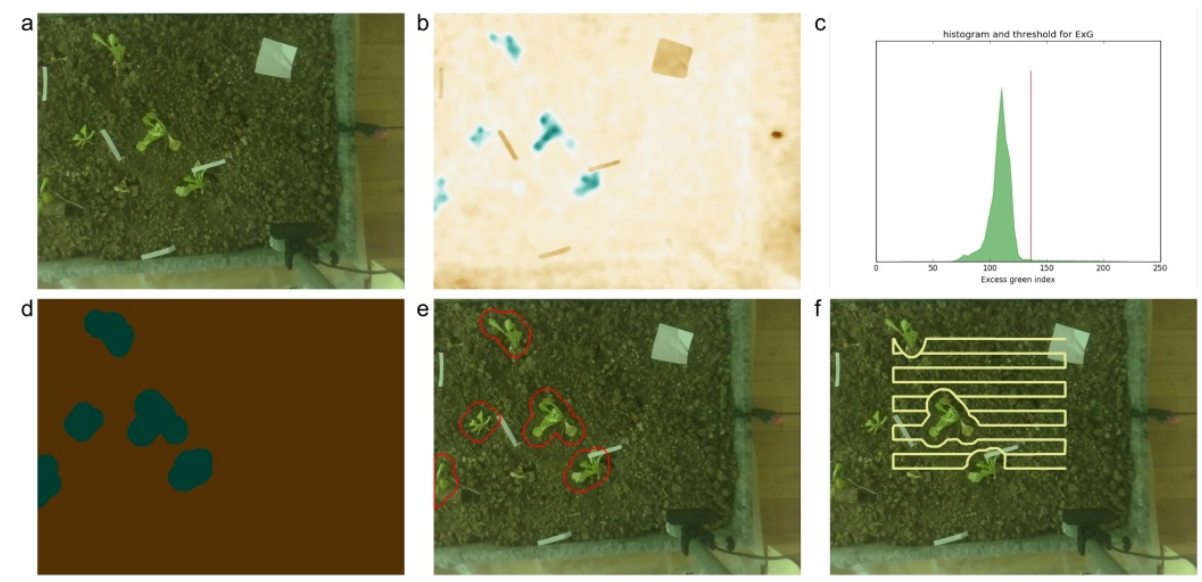

Figure 5. (a) Une caméra globale prend l'image du plan de travail qui se trouve sous le robot. (b) Pour trouver l'emplacement des plantes, l'algorithme calcule 


\section{Peter HANAPPE}

d'abord un histogramme des pixels verts dans l'image. (c) Sur la base de cet histogramme, un seuil est déterminé. (d) Ce seuil permet de segmenter l'image (e) et de tracer les contours des plantes. (f) À partir des contours, les trajectoires de l'outil sont calculées (Colliaux et Hanappe, 2017).

Le robot est facile à construire soi-même et relativement peu cher $^{5}$. Pour l'instant, l'outil ameublit la couche superficielle de la terre pour perturber la germination des mauvaises herbes. Dans une prochaine version, le robot pourra faire une classification des plantes pour distinguer les adventices (ou les mauvaises herbes) des cultivars et faire un enlèvement sélectif. D'autres tâches sont aussi envisageables, comme planter les graines ou repiquer les jeunes plants. Éventuellement, nous pourrions adapter le robot pour travailler sur un sol couvert (engrais verts ou paillage), ce qui réduit les risques d'érosion du sol et diminue les besoins en arrosage. Nous escomptons que ce type de robot offrira plusieurs avantages. Son faible poids évite le compactage du sol. II offre la possibilité de gérer des polycultures dans des géométries variables. Le désherbage mécanique élimine le besoin en pesticides et réduit le travail manuel pénible. II demande moins d'énergie que les machines conventionnelles ${ }^{6}$. Le robot permet une plus grande densité de cultures, donc un rendement plus important que les techniques conventionnelles. Nous évaluons également l'utilisation des caméras 3D (caméras "time-of-flight») pour observer les plants au cours du temps et extraire des indices de croissance (volume, surface foliaire) (Colliaux, Wintz et Hanappe, 2017).

\section{La gouvernance des nouvelles technologies}

Le développement de ce robot est une activité passionnante, néanmoins nous ne sommes pas insensibles aux critiques sur les outils numériques. Dans le livre TechNO-Fix, Huesemann et Huesemann (2011) font un constat accablant des effets néfastes des technologies. Dans l'agriculture, les techniques introduites

\footnotetext{
${ }^{5}$ Le coût des prototypes est moins de 5000 euros.

${ }^{6}$ Nous estimons qu'il est possible de faire fonctionner le robot avec un panneau solaire comme source d'énergie.
} 
Le permaculteur et son robot :

les microfermes et la gouvernance des nouvelles technologies

durant l'après-guerre sont aujourd'hui remises en question à cause de leurs externalités négatives. Un des problèmes de la situation actuelle est le modèle socioéconomique sous-jacent, notamment la concentration grandissante dans l'industrie agroalimentaire. Comme le rappellent Huesemann et Huesemann, les technologies sont souvent introduites pour résoudre des problèmes qui sont en fait sociétaux et qu'il faudrait aborder en changeant notre organisation et notre comportement. Effectivement, pour contrer le problème de la concentration économique, on pourrait développer les fermes familiales et agroécologiques qui optent pour une production locale, combinée avec une logistique décentralisée. C'est la solution qui est défendue entre autres par Fermes d'Avenir (2016) et Open Food Network (Bouré, 2015 ; Kurnia et al., 2015).

Peut-être que la réorganisation proposée par les microfermes en permaculture suffit pour résoudre les problèmes de l'agriculture industrielle. La question suivante se pose alors : s'il est possible de faire de l'agroécologie sans outils numériques, faut-il d'emblée les laisser de côté ? De même, si nous ne connaissons actuellement pas l'impact de ces outils sur le long-terme, faut-il les interdire ? Bien que nous comprenions les craintes que certains peuvent avoir, la position du rejet des technologies nous semble vouloir éviter tout débat, tout comme certaines réponses du camp protechnologie manquent de profondeur: « il faut développer les nouvelles technologies parce qu'on peut, parce que ça rapporte, ou parce que c'est amusant... ». On ne peut ignorer que les outils robotiques existeront, alors pourquoi ne pas démarrer la réflexion sur leur conception et leur gouvernance dès aujourd'hui ? Que faut-il mettre en place, aujourd'hui, pour s'assurer que l'application de ces outils soit " positive " ? Entre le rejet de la technologie d'un côté et l'adoption indiscriminée de nouvelles technologies de l'autre côté, il existe à notre avis une troisième option: le développement de ces technologies en tant que bien commun. Voyons d'abord quels sont les craintes et les risques de la situation actuelle. 


\section{Peter HANAPPE}

\subsection{Les objections}

Une partie des agriculteurs redoutent que les spécifications des outils soient écrites sans leur consultation et que ces outils profitent plus aux industriels qu'à eux-mêmes (InPACT, 2016). Ils craignent une perte d'autonomie et que les technologies créent une nouvelle forme de dépendance aux fournisseurs industriels, ce que Heather Marsh appelle un "monetized system of dissociation" (Marsh, 2013 ; voir aussi Wiens, 2015). Dans le rapport Bournigal et al. (2015), les auteurs admettent implicitement qu'il peut y avoir un problème. Ils mettent en avant que le fait de dépendre des services numériques étrangers est une menace pour la production agricole française. Or, pour un agriculteur, dépendre des services d'une start-up, même si elle est française, n'est pas rassurant non plus.

Plusieurs agriculteurs, avec qui nous avons pu discuter, ont exprimé la crainte que la robotique les éloigne de la terre et que cela réduise leurs observations de terrain. II existe aussi le risque que la robotique mène vers une nouvelle forme de normalisation des pratiques au moment où une diversité de modèles agricoles se développe (Petit, 2013) et où l'agroécologie plaide pour une diversification et une attention particulière aux fonctions écologiques uniques à chaque site. On craint que la robotique mène vers une nouvelle forme de productivisme et réduise encore plus le nombre d'emplois. D'autres points sont encore à noter : les questions sur la propriété des données des capteurs, sur la consommation d'énergie des infrastructures TIC et sur le recyclage des circuits électroniques (Bihouix, 2014).

Finalement, comme l'ont décrit Morel et Léger (2015), les aspirations des maraîchers vivriers vont au-delà du revenu et de la productivité. Ils cherchent aussi l'autonomie et ils veulent s'assurer que leur travail ait un sens (voir aussi d'Allens et Leclair, 2016). Cette envie de proximité avec la nature semble en contradiction avec une agriculture automatisée. 
Le permaculteur et son robot :

les microfermes et la gouvernance des nouvelles technologies

\subsection{Les licences libres comme garantie}

Nous publions les schémas et le code source de notre robot sous des licences libres ${ }^{7}$. Ces licences permettent aux agriculteurs de le construire eux-mêmes, de le modifier et de partager leurs modifications (Stallman, 1996). Ils pourront s'approprier le design pour l'adapter à leurs besoins. Ainsi, ils pourront concevoir le robot en gardant les tâches les plus intéressantes pour eux, les tâches qui valorisent leur savoir-faire, tandis que les outils intelligents seront là pour les assister sous leur contrôle et pour limiter les tâches pénibles, répétitives ou inintéressantes. Ils seront libres de choisir leur formule d'hébergement pour stocker les données. Comme effet de bord, nous espérons que les outils intelligents accessibles et modifiables permettront une démocratisation des équipements agricoles et faciliteront les installations des «néopaysans $»^{8}$.

Pour garantir cette liberté, il faut d'abord que toutes les couches logicielles du robot soient libres, ainsi que les données nécessaires à leur création - notamment les données pour l'apprentissage des modules d'intelligence artificielle ${ }^{9}$. De plus, la conception du robot doit aller de pair avec un projet de documentation poussé. Au-delà de ces questions techniques sur l'accès au code source et aux données, il y a un aspect social qui doit être pris en compte.

\subsection{Un commun des pratiques agricoles}

L'agriculture est une composante clé dans la transition vers une société durable. Elle engage donc tout le monde et notamment les citoyens. Nous avons déjà vu que les consommateurs demandent

\footnotetext{
${ }^{7}$ Le code source et les plans seront disponibles à :

https://github.com/romi. Voir aussi: https://p2pfoodlab.net et http://romi.farm.

${ }^{8}$ En 2004 , plus de $30 \%$ des nouvelles installations étaient " hors cadre familial », c'està-dire que le nouvel exploitant n'avait pas de famille proche (parents, grands-parents...) issue du milieu agricole (Lefebvre et Quelen, 2004).

${ }^{9}$ Quelques exemples : 1) une grande partie du système d'exploitation Android est libre, mais les modules non libres combinés à un chiffrement empêchent une autonomie aux utilisateurs ; 2) de plus en plus de logiciels libres dépendent de services web non libres ; 3 ) les algorithmes pour développer des applications en intelligence artificielle sont libres, mais demandent souvent une grande quantité de données pour l'entraînement qui ne le sont pas.
} 


\section{Peter HANAPPE}

plus de transparence sur les origines et les modes de production des aliments. Prenons par exemple la production des légumes dans les fermes "indoor », ou encore les "fermes-usines ». Cette technique peut sûrement valoriser des espaces urbains perdus, comme les garages vides ${ }^{10}$, mais des voix se lèveront sûrement si on transforme des terres fertiles en usines à légumes, même si la technologie utilisée est en libre accès. Ensuite, prédire en amont les impacts d'une technologie est quasi impossible, car ces effets deviennent visibles seulement après une utilisation à grande échelle et pendant une durée suffisante. II faut donc instaurer une évaluation continue qui implique surtout les agriculteurs, leurs voisins et les consommateurs.

Nous pensons que le système des labels participatifs offre le cadre que nous cherchons pour la gouvernance des outils numériques. En France, nous retrouvons, par exemple, le label « Nature \& Progrès », qui a été créé au début des années 1970 pour préserver des pratiques paysannes et biologiques. Ce groupement de producteurs et de consommateurs a défini des cahiers des charges qui décrivent les pratiques conseillées et a mis en place un système participatif de garantie pour délivrer les certifications. La fédération utilise un logo placé sur les emballages pour que les consommateurs puissent clairement identifier ce qui est produit dans le respect des engagements du label. La particularité de cette organisation est que l'ensemble des pratiques de production et de transformation est géré comme un bien commun. Le système d'évaluation mis en place répond aux huit principes caractéristiques des biens communs décrits par Ostrom (1990). Dans le cas de Nature \& Progrès, l'objectif de ce commun est «la pratique de l'agriculture biologique et le respect de l'environnement dans leurs aspects sociaux, techniques et économiques » (Fédération Nature \& Progrès, 2013b). La communauté est constituée des consommateurs et des professionnels adhérents de l'association. Le Système participatif de garantie, avec ses "commissions mixtes d'agrément et de contrôle locales ", assure la cohérence entre les règles relatives à la ressource commune et les conditions locales. En particulier,

\footnotetext{
${ }^{10}$ Voir : http://www.parisculteurs.paris/fr/sites/1339-parking-raymond-queneau-18e.html.
} 
Le permaculteur et son robot :

les microfermes et la gouvernance des nouvelles technologies

celle-ci est assurée par la gradation des sanctions pour nonrespect des règles et le fait que ce sont les exploitants et les consommateurs eux-mêmes qui surveillent les pratiques et qui en rendent compte aux autres agriculteurs. II existe des comités techniques internes à travers lesquels les membres participent à la modification des règles concernant la ressource commune (Fédération Nature \& Progrès, 2013a, 2013b). II est important d'ajouter que d'autres outils existent pour inclure les citoyens dans les débats sur les impacts technologiques, nous pensons particulièrement aux procédures dites " convention de citoyens " (Testart et al., 2007). Ces procédures sont une inspiration, de manière générale, sur comment structurer les discussions dans les comités techniques.

En prenant l'exemple de Nature \& Progrès, nous voulons montrer qu'en plus d'un bien commun informationnel (logiciels libres, open hardware, open data), il est important d'instaurer un bien commun des pratiques agricoles. L'objectif de ce commun serait: (1) de trouver un juste milieu entre un techno-optimisme d'un côté et un rejet des nouvelles technologies de l'autre côté ; (2) d'assurer que les outils robotiques et l'intelligence artificielle s'inscrivent dans une approche agroécologique forte avec des pratiques durables sur le plan environnemental et socioéconomique ; et (3) de valoriser l'intervention humaine.

Même si les outils numériques seront réalisés en bonne partie par des entreprises et par des centres de recherches (publics et privés), il est crucial de mettre en place une gouvernance qui implique ceux qui sont les plus concernés par ces nouvelles pratiques: les agriculteurs et les citoyens informés. Ce sont ces deux groupes qui ont le plus besoin de se faire entendre et de se structurer. Sans leur participation, le risque que les TIC soient imposées par des tiers reste réel. Nous proposons donc d'élargir l'objectif des labels participatifs pour y intégrer la question de l'agriculture numérique et de mettre en place des groupes de travail mixtes pour œuvrer sur un cahier des charges de la robotique, de l'intelligence artificielle, des TIC et de l'exploitation des données. Les "tiers-lieux ${ }^{11}$ " comme les fab labs déjà cités

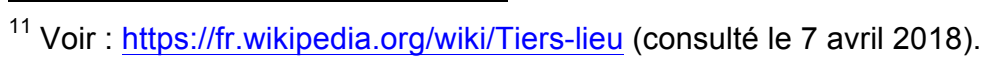




\section{Peter HANAPPE}

peuvent être des lieux où les acteurs intéressés par un tel développement puissent se rencontrer. Les labels pourraient être vecteurs d'informations plus précises encore. Une agriculture durable comprend beaucoup de facettes : réduction des intrants, rationalisation de la consommation d'eau, bien-être des animaux, santé des sols, biodiversité, vente en circuit court... Ces différents critères pourraient donner lieu à des "labels thématiques", toujours attribués via un système participatif de garantie. Pour leurs achats, les consommateurs pourront ainsi appliquer leur propre grille d'évaluation. Une personne soucieuse des émissions $\mathrm{CO}_{2}$ pourra par exemple favoriser un producteur qui utilise des énergies renouvelables. Les TIC pourront faciliter l'accès à ces informations détaillées.

\section{Discussion}

Nous avons présenté notre projet robotique pour les microfermes agroécologiques. À plus long terme, nous imaginons ce robot comme une plateforme flexible qui pourra faire des interventions précises et assister l'agriculteur dans le suivi et la planification des cultures. Bien que nous ne soyons qu'au tout début du projet, les possibilités de combiner cette plateforme flexible avec les TIC pour la transmission et le partage des informations et avec les techniques d'intelligence artificielle pour un apprentissage continu sont nombreuses. II n'est pas difficile d'envisager que ce type d'outils puisse avoir un impact à long terme sur l'agriculture.

Notre objectif, avec ce texte, est d'apporter une pierre à la discussion sur l'agriculture de demain - vaste chantier -, car nous sommes conscients que les nouvelles technologies peuvent engendrer des changements sociologiques et environnementaux imprévus et qu'elles ne sont pas séparées de la politique. Pour cette raison, nous avons voulu anticiper le débat en proposant une solution qui inscrit l'utilisation de ces technologies dans un modèle de gouvernance participatif, associé à un label de garantie pour les consommateurs. Nous pensons que cette démarche permettra de rapprocher deux mondes fascinants: les microfermes et la robotique. 
Le permaculteur et son robot :

les microfermes et la gouvernance des nouvelles technologies

Sous notre démarche réside une vision du système alimentaire qui est sans doute un peu idéaliste, mais dont les bases existent déjà, et qu'il serait utile d'expliciter.

1) Système alimentaire durable. Tout d'abord, l'objectif principal de notre démarche doit être de créer un système alimentaire durable, aussi bien sur le plan écologique que sur les plans social et économique. Les autres points affichés ci-dessous découlent de cet impératif.

2) Agroécologie. Laissons faire ce que la nature sait faire de mieux et utilisons au mieux les processus naturels pour créer un environnement productif optimal. L'approche agroécologique ainsi que les principes de la permaculture selon Holmgren semblent être les pistes à suivre (Gliessman, 2014 ; Holmgren, 2002). II faudra néanmoins réfléchir à la façon d'impliquer aussi les citoyens dans cette démarche (Hanappe, 2016).

3) Microfermes agroécologiques et distribution décentralisée. Les microfermes sont à notre avis un socle important pour parvenir à une agroécologie forte. Mises à part leur taille humaine et leur attention aux pratiques écologiques, ce qui nous intéresse également est leur modèle économique. Ces petites structures favorisent une organisation décentralisée et une économie de proximité. Personne ne comprenait cet aspect mieux que l'économiste Schumacher (1973): «Nous avons besoin de la liberté de beaucoup, beaucoup d'unités autonomes et de petite taille et, en même temps, la discipline de l'unité et de la coordination à grande échelle, voire globale ${ }^{12}$." (p. 49.) Les microfermes sont les unités autonomes: les TIC permettent la coordination à grande échelle, notamment pour la logistique de la distribution (Bouré, 2015 ; Kurnia et al., 2015).

4) Commun informationnel. Les technologies et les outils utilisés - qu'ils soient low-tech ou high-tech - doivent constituer un bien commun informationnel, être disponibles sous des licences libres, être réalisables par une personne amateur éclairée et être

\footnotetext{
12 Traduction libre de : "We need the freedom of lots and lots of small, autonomous units, and, at the same time, the orderliness of large-scale, possibly global, unity and coordination. ".
} 


\section{Peter HANAPPE}

utilisables par tous. Une fois de plus, nous nous appuyons sur les mots de Schumacher, qui a introduit la notion de technologie appropriée. II fait la distinction entre les technologies pour la production de masse, d'un côté, et les technologies pour la production par la masse, de l'autre côté. Ces dernières sont des technologies accessibles, conçues pour servir l'homme, et propices à des organisations décentralisées.

5) Commun des pratiques. Les pratiques constituent elles aussi un bien commun, comme nous l'avons souligné. Nous avons argumenté la mise en place d'une gouvernance qui inclut toutes les parties concernées, en premier lieu les agriculteurs et les consommateurs. Nous avons montré qu'un bien commun des pratiques avec un système d'évaluation participatif et local est une approche souhaitable pour encadrer le développement et l'introduction des TIC et de la robotique pour les microfermes. II faudra mettre en place une forme de "technoécologie »: la première loi de l'écologie de Barry Commonor - «tout est connecté à tout » - vaut aussi pour les technologies. L'utilisation des machines intelligentes créera de nouvelles interactions Nature-Homme-Machine qu'il faudra étudier avec les concepts mis en place par l'écologie en tant que discipline scientifique.

L'idée de combiner la permaculture avec la robotique est sans doute déjà provocatrice. Nous y rajoutons une couche en suggérant qu'il faudrait accélérer cette recherche et accentuer l'effort de développement des nouvelles technologies pour les microfermes. Notre raisonnement est que plus la communauté des agriculteurs agroécologiques définit les axes de recherche en amont, plus elle aura une voix dans les discussions sur l'agriculture de demain (voir Williams et Srnicek, 2013).

\section{Références}

Altieri, M. A. (1995). Agroecology: The Science Of Sustainable Agriculture $\left(2^{\mathrm{e}}\right.$ éd.). Boulder, CO : Westview Press Inc.

Aubry, C. et Chiffoleau, Y. (2009). Le développement des circuits courts et l'agriculture périurbaine : histoire, évolution en cours et questions actuelles. Innovations Agronomiques, 5, 53-67. 
Le permaculteur et son robot:

les microfermes et la gouvernance des nouvelles technologies

Bihouix, P. (2014). L'Âge des low tech. Paris, France: Seuil.

Bouanchaud, C. (2016, 23 août) Combien coûte la production d'un litre de lait. Le Monde. Repéré à http://www.lemonde.fr/lesdecodeurs/article/2016/08/23/combien-coute-la-production-d-unlitre-de-lait_4986851_4355770.html

Bouré, M. (2015, 6 novembre). Toward an age of agrocommunities: The third food revolution. OuiShare Magazine. Repéré à https://www.ouishare.net/article/toward-an-age-ofagro-communities-the-third-food-revolution

Bournigal, J.-M, Houllier, F., Lecouvey, P. et Pringuet, P. (2015). 30 projets pour une agriculture compétitive \& respectueuse de l'environnement (\#Agriculturelnnovation2025). Paris, France: Ministère de l'Agriculture, de l'Agroalimentaire et de la Forêt.

Carson, R. (1962). Silent Spring. Boston, MA : Houghton Mifflin Harcourt.

Charroin, T., Veysset, P., Devienne, S., Fromont, J.-L., Palazon, R. et Ferrand, M. (2012). Productivité du travail et économie en élevages d'herbivores: définition des concepts, analyse et enjeux. INRA Productions Animales, 25(2), 193-210.

Coleman, E. (1995). The New Organic Grower. White River Junction, VT : Chelsea Green Publishing.

Colliaux, D. et Hanappe, P. (2017). LettuceThink: A open and versatile robotic platform for weeding and crop monitoring on microfarms. Communication présentée à la conférence EFITA WCCA 2017, Montpellier, France.

Colliaux, D., Wintz, T., et Hanappe, P. (2017). Bringing phenotyping to the farm: An evaluation of $3 D$ reconstruction of plants in outdoor environment. Communication présentée à I'International Conference on Computer Vision, Venise, Italie.

Council of Economic Advisers (2016). Benefits of Competition and Indicators of Market Power. Washington, D.C. : White House.

d'Allens, G. et Leclair, L. (2016). Les néo-paysans. Paris, France: Édition du Seuil. 


\section{Peter HANAPPE}

De Schutter, O. (2014). Rapport final: le droit à l'alimentation, facteur de changement. New York, NY: UN Human Rights Council.

Food and Agriculture Organization of the United Nations (FAO) et Intergovernmental Technical Panel on Soils (ITPS) (2015). Status of the World's Soil Resources (SWSR) - Technical Summary. Rome, Italie : FAO et ITPS.

Fédération Nature \& Progrès (2013a). Charte. Alès, France: Fédération internationale " Nature \& Progrès ».

Fédération Nature \& Progrès (2013b). Statuts. Alès, France: Fédération internationale « Nature \& Progrès ».

Fermes d'Avenir (2016). Plaidoyer pour une agriculture innovante à impacts positifs. Montlouis-sur-Loire, France: Fermes d'Avenir.

Fortier, J.-M. (2012). Le jardinier-maraîcher: manuel d'agriculture biologique sur petite surface. Montréal, Québec : Écosociété.

Fuglie, K., Heisey, P. W., King, J. et Schimmelpfennig, D. E. (2012). Rising Concentration in Agricultural Input Industries Influences New Farm Technologies. Amber Waves, 10(4).

Garlanda, L. (2017). Évaluation d'un outil robotique pour le désherbage et le suivi des cultures bio-intensives (mémoire de Master). Agro-Campus Ouest et AgroParisTech, Paris.

Gliessman, S. R. (2014). Agroecology: The Ecology of Sustainable Food Systems ( $3^{\mathrm{e}}$ éd.). Boca Raton, FL : CRC Press.

Gonzalez-de-Santos, P. et al. (2016). Fleets of robots for environmentally-safe pest control in agriculture. Precision Agriculture, 18(4), 574-614.

Guégan, S. et Léger, F. (2015). Étude "Maraîchage biologique permaculturel et performance économique " - Rapport final. Paris, France : Institut Sylva et AgroParisTech - UMR SADAPT.

Hanappe, P., R. Dunlop, A. Maes, L. Steels et N. Duval (2016). Agroecology: A fertile ground for Human Computation. Human Computation Journal, 3(1), 225-233. 
Le permaculteur et son robot:

les microfermes et la gouvernance des nouvelles technologies

Hervé-Gruyer, C. (2015). Recherche d'outils innovants en faveur de la micro-agriculture permaculturelle. Bec Hellouin, France: La Ferme du Bac Hellouin.

Hervé-Gruyer, P. et Hervé-Gruyer, C. (2014). Permaculture, Guérir la Terre, nourrir les Hommes. Paris, France : Actes Sud.

Hess, C. et Ostrom, E. (2011). Understanding Knowledge as a Commons, From Theory to Practice. Cambridge, MA: MIT Press.

Holmgren, D. (2002). Permaculture Principles and Pathways Beyond Sustainability. East Meon, Angleterre: Permanent Publications.

Holt-Giménez, E. (2006). Campesino A Campesino: Voices from Latin America's Farmer to Farmer Movement for Sustainable Agriculture. Oakland, CA : Food First Books.

Huesemann, M. et Huesemann, J. (2011). Techno-Fix: Why Technology Won't Save Us Or the Environment. Gabriola Island, Colombie-Britannique : New Society Publishers.

International Federation of Organic Agriculture Movements (IFOAM) (2008). Les Systèmes de garantie participatifs. Bonn, Allemagne : IFOAM.

InPACT (2016). Souveraineté technologique des paysans: défendre l'intérêt général autour des agroéquipements. Paris, France : InPACT.

Jeavons, J. (2012). How to Grow More Vegetables (and Fruits, Nuts, Berries, Grains, and Other Crops) Than You Ever Thought Possible on Less Land Than You Can Imagine ( $8^{\mathrm{e}}$ éd.). Berkeley, CA : Ten Speed Press.

Kurnia, S., Hill, S., Rahim, M. M., Larsen, K., Braun, P. et Samson, D. (2015). Open Food Network: The role of ICT to support regional food supply chains in Australia. Communication présentée à la $26^{\text {th }}$ Australasian Conference on Information System, Adelaide, Australie.

Lefebvre, F. et Quelen, M. (2004). Le devenir des agriculteurs installés Hors du cadre familial: le renouvellement des 


\section{Peter HANAPPE}

générations agricoles bientôt assure par des citadins?. Limoges, France : CNASEA.

Lowder S. K, J. Skoet et S. Singh (2014). What do we really know about the number and distribution of farms and family farms in the world? Background paper for The State of Food and Agriculture 2014. Rome, Italie: Rome Food et Agriculture Organization of the United Nations.

Marsh, H. (2013, 24 mais). Binding Chaos. Heather Marsh. Rethinking the moats and mountains. Repéré à https://georgiebc.wordpress.com/2013/05/24/binding-chaos/

Mollison, B. et Holmgren, D. (1990). Permaculture One: A Perennial Agricultural System for Human Settlements ( $5^{e}$ éd.). Sisters Creek, Australie : Tagari Publications.

Morel, K. et Léger, F. (2015). Comment aborder les choix stratégiques des paysans alternatifs? Le cas des microfermes maraîchères biologiques en France. Paris, France: AgroParisTech - UMR SADAPT.

Mulla, D. J. (2013). Twenty five years of remote sensing in precision agriculture: Key advances and remaining knowledge gaps. BioSystems Engineering, 114(4), 358-371.

Ostrom, E. (1990). Governing the Commons: The Evolution of Institutions for Collective Action. Cambridge, Angleterre: Cambridge University Press.

Pereira, M. A., Kartashov, A. I., Ebbeling, C. B., Van Horn, L., Slattery, M. L., Jacobs Jr, D. R. et Ludwig, D. S. (2005). Fastfood habits, weight gain, and insulin resistance (the CARDIA study): 15-year prospective analysis. The Lancet, 365(9453), 36-42.

Petit, S. (2013). Faut-il absolument innover? À la recherche d'une agriculture d'avant-garde. Communication présentée aux VIII ${ }^{\text {es }}$ Rencontres des exploitations et ateliers technologiques de l'enseignement agricole, Obernai, France. 
Le permaculteur et son robot:

les microfermes et la gouvernance des nouvelles technologies

Pimentel, D. (2006). Soil Erosion: A Food and Environmental Threat. Environment, Development and Sustainability, 8(1), 119137.

Schumacher, E. F. (1973). Small is Beautiful: A Study of Economics As If People Mattered. Londres, Angleterre : Blond \& Briggs.

Shand, H. (2012). The Big Six: A Profile of Corporate Power in Seeds, Agrochemicals \& Biotech. The Heritage Farm Companion, (Summer), 10-15.

Stallman, R. (1996). Qu'est-ce que le logiciel libre ? Définition du logiciel libre. Système d'exploitation GNU. Repéré à https://www.gnu.org/philosophy/free-sw.html

Testart J., Callon, M., Hermitte, M.-A. et Rousseau, D. (2007). Des Conférences de citoyens en droit français. Paris, France: Fondation Sciences citoyennes.

Transrural (2009). Dossier 383: les agricultures alternatives. Transrural initiatives, (382).

Wezel, A., Bellon, S., Dore, T., Francis, C., Vallod, D. et David, C. (2009). Agroecology as a science, a movement and a practice. A review, Agronomy for Sustainable Development, 29(4), 503515.

Wiens, K. (2015, 2 mai). New High-Tech Farm Equipment Is a Nightmare for Farmers. Wired. Repéré à

https://www.wired.com/2015/02/new-high-tech-farm-equipmentnightmare-farmers/

Williams, A. et N. Srnicek (2013, 14 mais). Accelerate Manifesto for an Accelerationist Politics, Critical Legal Thinking. Critical Thinking. Repéré à

http://criticallegalthinking.com/2013/05/14/accelerate-manifestofor-an-accelerationist-politics/ 